\title{
Mammalian Oocyte Quality in Relation to Proteome at Different Stages of Development: An Update
}

\author{
Raghvendra Kumar* and Prabhat Palta \\ Animal Biotechnology Centre, ICAR-National Dairy Research Institute, \\ Karnal, Haryana, India \\ *Corresponding author
}

\begin{tabular}{|l|l}
\hline \multicolumn{2}{c}{ A B S T R A C T } \\
\cline { 2 - 3 } $\begin{array}{l}\text { Keywords } \\
\begin{array}{l}\text { Oocyte, Proteome, } \\
\text { Oocyte quality, } \\
\text { Mass spectrometry, } \\
\text { Proteomic strategy }\end{array}\end{array}$ & $\begin{array}{l}\text { Mammalian oocytes are pivotal cells in reproduction, still the proteomic aspect } \\
\text { within oocyte held responsible for its development and behaviour are partly } \\
\text { pictured. Hence modern proteomic strategies have been employed largely to } \\
\text { provide new illustration of oocyte quality. High throughput proteomics have } \\
\text { unrevealed many insights in various stages of oocyte across the species. } \\
\text { Specific proteome studies conducted on oocyte evidenced towards complete } \\
\text { repertoire of protein set present in oocyte. The evidenced molecular qualities } \\
\text { Article Info }\end{array}$ \\
$\begin{array}{l}\text { Accepted: } \\
\text { 26 September 2020 in improving protocols of assisted reproduction. Present review aims to } \\
\text { Available Online: } \\
\text { 10 October 2020 }\end{array}$ & $\begin{array}{l}\text { accumulate animal oocyte findings in terms of protein research in oocyte } \\
\text { through available literature, which remain to be emphasized for oocyte } \\
\text { proteomics. }\end{array}$ \\
\hline \hline
\end{tabular}

\section{Introduction}

In the course of growth and maturation of oocyte, numerous maternal mRNA gets accumulated which eventually contribute to its developmental competence to get fertilized. Most of the mRNA among these forms complex with protein, and remain silent as ribonucleoprotein. Message ribonucleoprotein gets selectively translated according to need during specific stages of growth phase (Hafidh et al., 2011). The exact amount of protein expression in oocyte varies according to species and stage of oocyte which is still poorly understood. Although it is evidenced that regulation of translational activity is vital for development and maturation of oocyte (MacNicol et al., 2010).

High throughput proteomic strategies and powerful approaches have potential to deduce discrete aspect of developmental quality, but this domain needs more to be explored.

In recent past year's oocyte proteomic studies were conducted on different mammalian oocyte. Aim of the present review is to summarize these literatures of different species to an update for oocyte proteome. 


\section{Use of proteomics for studying oocytes}

The proteomics approach can be a very efficient means for elucidation of the highly complex processes which make an oocyte developmentally competent. Despite availability of powerful proteomics techniques in the last decade, very few studies have been conducted in oocytes for deciphering its proteome primarily due to non-availability of oocytes in numbers sufficient to carry out such studies. Among proteomics techniques, DIGE and cICAT are used in gel and LC-based quantitative proteomics respectively, whereas, iTRAQ is a new LC-based technique which is gradually gaining popularity. In DIGE, accurate quantification is sometimes compromised due to co-migration or partial co-migration of proteins. The iTRAQ method is more susceptible to errors in precursor ion isolation, which can be manifested with increasing sample complexity. The global-tagging iTRAQ technique is more sensitive than the cysteine specific cICAT method, which in turn is as sensitive as, if not more sensitive than, the DIGE technique.

\section{Proteomics related to oocyte quality}

In a study on the comparison of extracts of high and low quality porcine oocytes ExacTag ${ }^{\mathrm{TM}}$ proteomics technology, Powell et al., (2010) recorded a total of 503 significant protein IDs in the combined proteome of high (IVM in presence of gonadotropins) and low (IVM in absence of gonadotropins) quality pig oocytes. These authors identified 16 predominant putative oocyte quality marker proteins among which, more abundant proteins in the high-quality oocytes included kelch-like ECH-associated protein 1 (an adaptor for ubiquitin ligase CUL3), nuclear export factor CRM1 and ataxia telangiectasia mutated protein kinase. In the low-quality oocytes, dystrophin (DMD) was found to be more abundant. These authors identified 110 proteins, including DMD and cystic fibrosis transmembrane conductance regulator, two proteins implicated in muscular dystrophy and cystic fibrosis, respectively, in the secretome.

\section{Proteomics related to oocyte maturation}

A large percentage of immature oocytes fail to develop to the blastocyst stage indicating failure of oocyte maturation. Proteomic profiling of mice GV and MII-arrested oocytes carried out by Vitale et al., (2007) revealed 12 proteins differentially expressed between the GV and MII stages. These proteins included transforming acidic coiledcoil protein 3 (TACC3), heat shock protein 105 (HSP105), programmed cell death sixinteracting protein (PDCD6IP), stressinducible phosphoprotein (STI1), importin alpha 2, adenylsuccinate synthase (ADDS), nudix, spindlin, lipocalin, lysozyme, translationally controlled tumor protein (TCTP) and nucleoplasmin 2 (NPM2). These proteins which were identified using MS, were found to be related to apoptosis, stress and post-translational modifications. There are very few reports on the proteomics of oocytes in buffalo. In a recent study, Chen $e t$ al., (2016) investigated the proteomics of buffalo oocytes of different maturation stages and developmental competence status. These authors investigated the protein profile of buffalo oocytes at the GV and MII stage using iTRAQ. They identified a total of 3,763 proteins among which, 173 proteins were differentially expressed in GV oocytes and competent MII oocytes, and 146 proteins were differentially abundant in competent and incompetent matured oocytes. Functional and KEGG pathway analysis of the data revealed that the up-regulated proteins in competent MII oocytes were related to chromosome segregation, microtubule-based process, protein transport, oxidation reduction, ribosome, and oxidative phosphorylation, etc., 
in comparison with GV and incompetent MII oocytes. The authors suggested that some of these proteins may potentially act as markers to predict developmental competence of buffalo oocyte during in vitro maturation.

In a study aimed at examining the changes in proteins during oogenesis in the Swamp buffalo, Fu et al., (2016) subjected proteins from immature and in vitro matured oocytes to two-dimensional electrophoresis, and detected more than 400 spots. A total of 17 proteins were found to be differentially expressed between the two groups by image analysis. Eight proteins were identified by MS. In mature oocytes, the three proteins which were down-regulated included major vault protein (MVP), $\mathrm{N}$-acetyl lactosaminide1,6-Nacetylglucosaminyl-transferase (GCNT2 ), and gem-associated protein (GEMIN)8 whereas, five other proteins, which were upregulated included heat shock protein (HSP) 60, Ras-responsive element-binding protein 1 (RREB-1), heat shock cognate $71 \mathrm{kDa}$ protein (HSC71), hemoglobin subunit (HBA), and BMP-2-inducible protein kinase (BMP-2K). The authors further verified the expression profiles of HSP60 and GEMIN8 by Western blotting. The changes in HSP60 protein expression demonstrate the increasing need for mitochondrial protein import to facilitate macromolecular assembly during oocyte maturation. The down-regulation of GEMIN8 production implies that RNA splicing is impaired in mature oocytes. Virant-Klun et al., (2016) carried out the first proteome and secretome maps of human oocytes for understanding proteome composition and diversity during oocyte maturation. These authors identified maturation-specific proteins by single-cell proteomics of human oocyte. Starting from 100 oocytes collected via a novel serum-free hanging drop culture system, they identified 2,154 proteins, whose function indicate that oocytes are largely resting cells with a proteome that is tailored for homeostasis, cellular attachment, and interaction with its environment via secretory factors. These authors also identified 158 oocyte-enriched proteins such as ECAT1, PIWIL3, NLRP71 not observed in high coverage proteomics studies of other human cell lines or tissues. They scaled down the proteome analysis to single cells by exploiting SP3, a novel technology for proteomic sample preparation using magnetic beads. Despite the low protein content of only $100 \mathrm{ng}$ per cell, they consistently identified 450 proteins from individual oocytes. When comparing individual oocytes at the GV and MII stage, they found that the Tudor and $\mathrm{KH}$ domaincontaining protein (TDRKH) were preferentially expressed in immature oocytes, while Wee2, PCNA, and DNMT1 were enriched in mature cells. This indicated that maintenance of genome integrity is crucial during oocyte maturation. The study demonstrates that an innovative proteomics work flow facilitates analysis of single human oocytes to investigate oocyte biology and preimplantation development. The approach presented by them paves the way for quantitative proteomics in other quantitylimited tissues and cell types.

Recently a study in domestic cat (Feliscatus) using one dimentional electrophoresis LCMS method, (Turathum et al., 2020) reported qualitative profiles of protein among GV stage and MII stage. Out of 260 proteins analysed, 81 were differentially expressed among GV and MII stage. Further protein analysis revealed substantial expression involved in transportation which may indicates important characteristic present in GV. Proteome of MII oocytes showed over expression of cell cycle proteins. Notably, the author reported DNA repair and apoptosis related proteins were only expressed in GV stage, whereas proteins related to fertilization were only present in MII oocytes. 


\section{Proteomics of interaction between oocyte and cumulus cells}

The cumulus cells bind to zonapellucida, the outer covering of the oocyte and connect to the cytoplasmic membrane of the oocyte to form a COC through transzonal cytoplasmic process called gap junctions which permit exchange of small molecules between the oocyte and the cumulus cells (Albertini et al., 2001). Although this bidirectional communication and paracrine signaling between the cumulus cells and the oocytes are critical for making the oocyte developmentally competent, (Eppig et al., 1993, De La Fuenta and Eppig 2001, Gilchrist et al., 2003, Sugiura and Eppig 2005), the nature of this communication and its effects on the transcriptome and proteome of both cumulus cells and oocyte are poorly understood. First comprehensive proteome analysis of bovine $\mathrm{GV}$ stage oocytes and cumulus cells, including membrane proteins, reported that 4395 and 1092 proteins were expressed in the cumulus cells and the oocytes, respectively (Memili et al., 2007). Further, 858 proteins were common to both the cumulus cells and the oocytes. These proteins not only provide a foundation for signaling and cell physiology at the GV stage of oocyte development, but are also valuable for comparative studies of other stages of oocyte development at the molecular level. Some of these proteins may be potential biomarkers for developmental competence of oocytes. In another study, Peddinti et al., (2010) used differential detergent fractionation multidimensional protein identification technology (DDF-Mud PIT) for elucidating the proteome of bovine GV oocytes and cumulus cells. They identified 811 and 1247 proteins in GV oocyte and cumulus cells, respectively, among which 371 proteins were significantly differentially expressed between each cell type. Systems biology modeling of bovine oocytes and cumulus cells in the context of gene ontology and protein interaction networks identified the signaling pathways associated with the proteins involved in cell-to-cell signaling biological process that may have implications in oocyte competence and maturation.

\section{Proteomics in zona free oocyte}

Zhang et al., (2009) used high throughput proteomics approach for identification of maternal proteins in zona-free mouse oocytes for identification of proteins in cytoplasm having role in development. The authors reported the presence of 625 different proteins among which, 76 maternal proteins were observed to have high levels of mRNA expression in both oocytes and fertilized eggs. Many well-known maternal effect proteins were included in this subset. These included MATER, in the absence of which, embryonic development does not proceed beyond the 2cell stage and NPM2, which is essential for nuclear and nucleolar organization during embryonic development. Cao et al., (2012) compared proteomic profiles between $\mathrm{GV}$ and MII mouse oocytes (zona free) by twodimensional electrophoresis and identified 95 differentially expressed protein spots corresponding to 63 proteins. Many of these proteins such as adenylosuccinate synthetase, nucleoplasmin-2, and protein-arginine deiminase type 6 are known to be essential for oocyte meiosis and early embryonic development. Of the 12 proteins that were identified and were highly expressed in oocytes, a novel protein, E330034G19Rik, was found to be oocyte-specific. This protein may be involved in regulation of chromosome segregation during meiosis or cleavage.

\section{Proteomics at different developmental stage of oocyte}

Bioinformatics analysis of the proteome of mouse oocytes at different stages of 
development showed different protein composition in correlation with oocyte characteristics e.g., specific transcription factors and chromatin remodeling factors, which may be crucial for the epigenetic reprogramming of sperm or somatic nuclei (Wang et al., 2010). The authors identified 2,781 proteins present in GV-stage oocytes, 2,973 proteins in MII-stage oocytes and 2,082 proteins in zygotes through semi quantitative MS analysis. Ubiquitination pathway was found to be altered significantly in zygotes after fertilization, as the maternal proteins are quickly degraded. As a result, less number of peptides were identified in zygotes as compared to MII oocytes although equal number of oocytes and zygotes were analyzed. These results showed that the proteins involved in the ubiquitination pathway are highly enriched in the zygote, where they may play essential roles in degrading the maternal proteins inherited from the MII oocytes. This study provided a deep insight into the protein expression profile of mouse oocytes and demonstrated that oocytes are quite "active" cells expressing proteins related to a range of biological functions such as protein metabolism, transport, cell cycle and proliferation, stress response, developmental processes, RNA and DNA metabolism, cell organization and biogenesis, cell-cell signaling, signal transduction, and cell adhesion. By using a TMT labeled quantitative proteomics in combination with RNA sequencing strategy eight (GV, MII, 2cell, 4-cell, 8-cell, 16-cell, morula, blastocyst) stages transcriptome was analysed (Chen et al., 2018). These author sidentified a total of 3567 unique genes differently expressed among all consecutive stages of preimplantation development.

In conclusion the proteomic based information available in different vertebrate species point to substantial similarities in the regulatory pathways and mechanisms related to oocyte development. Relatively few subset of protein differs in expression during various stages of development. This robust differential expression needs continuity for exploration. Of the several models and methods used in these studies, each model provides unique information that could help decipher the oocyte competence and elucidate how oocytes control it. Exploration through sensitive proteomic approaches could be related in perspectives of human oocyte. In addition, these studies may provide valuable information on the growth and development of oocytes. Robust proteomic strategy can identify biomarker for effective oocyte selection. Through basic research many data sets have been produced but may be less clinical. Thus it needs to translate these insights in clinical applications.

\section{References}

Albertini, D.F., Combelles, C.M., Benecchi, E. and Carabatsos, M.J., 2001. Cellular basis for paracrine regulation of ovarian follicle development. Reproduction, 121(5): 647653.

Cao, S., Guo, X., Zhou, Z. and Sha, J., 2012.Comparative proteomic analysis of proteins involved in oocyte meiotic maturation in mice. Molecular reproduction and development, 79(6): 413-422.

Chen, F., Fu, Q., Pu, L., Zhang, P., Huang, Y., Hou, Z., Xu, Z., Chen, D., Huang, F., Deng, T. and Liang, X., 2018. Integrated analysis of quantitative proteome and transcriptional profiles reveals the dynamic function of maternally expressed proteins after parthenogenetic activation of buffalo oocyte. Molecular \& Cellular Proteomics, 17(10): 1875-1891.

De La Fuente, R. and Eppig, J.J., 2001. Transcriptional activity of the mouse oocyte genome: companion granulosa cells modulate transcription and chromatin remodeling. Developmental biology, 229(1): 224-236. 
Eppig, J.J., Wigglesworth, K. and Chesnel, F., 1993. Secretion of cumulus expansion enabling factor by mouse oocytes: relationship to oocyte growth and competence to resume meiosis. Developmental biology, 158(2): 400-409.

Fu, Q., Liu, Z.F., Huang, Y.L., Lu, Y.Q. and Zhang, M., 2016. Comparative proteomic analysis of mature and immature oocytes of the swamp buffalo (Bubalusbubalis). International journal of molecular sciences, 17(1): 94.

Gilchrist, R.B., Morrissey, M.P., Ritter, L.J. and Armstrong, D.T., 2003. Comparison of oocyte factors and transforming growth factor- $\beta$ in the regulation of DNA synthesis in bovine granulosa cells. Molecular and cellular endocrinology, 201(1-2): 87-95.

Hafidh, S., Čapková, V. and Honys, D., 2011. Safe keeping the message: mRNP complexes tweaking after transcription. In RNA infrastructure and Networks (118136).Springer, New York, NY.

MacNicol, M.C. and MacNicol, A.M., 2010. Developmental timing of mRNA translation integration of distinct regulatory elements. Molecular reproduction and development, 77(8): 662-669.

Memili, E., Peddinti, D., Shack, L.A., Nanduri, B., McCarthy, F., Sagirkaya, H. and Burgess, S.C., 2007. Bovine germinal vesicle oocyte and cumulus cell proteomics. Reproduction, 133(6): 11071120.

Peddinti, D., Memili, E. and Burgess, S.C., 2010. Proteomics-based systems biology modeling of bovine germinal vesicle stage oocyte and cumulus cell interaction. Plos one, 5(6): 11240.

Powell, M.D., Manandhar, G., Spate, L.,
Sutovsky, M., Zimmerman, S., Sachdev, S.C., Hannink, M., Prather, R.S. and Sutovsky, P., 2010.Discovery of putative oocyte quality markers by comparative ExacTag proteomics. PROTEOMICSClinical Applications, 4(3): 337-351.

Sugiura, K. and Eppig, J.J., 2005. Society for Reproductive Biology Founders' Lecture 2005. Control of metabolic cooperativity between oocytes and their companion granulosa cells by mouse oocytes. Reproduction, Fertility and Development, 17(7): 667-674.

Turathum, B., Saikhun, K., Roytrakul, S., Changsangfa, C., Tanasawet, S., Sroyraya, M. and Kitiyanant, Y., 2020. Comparative proteomic analysis of mature and immature oocytes in domestic cats. Asian Pacific Journal of Reproduction, 9(1): 22.

Virant-Klun, I., Leicht, S., Hughes, C. and Krijgsveld, J., 2016.Identification of maturation-specific proteins by single-cell proteomics of human oocytes. Molecular \& Cellular Proteomics, 15(8): 2616-2627.

Vitale, A.M., Calvert, M.E.K., Mallavarapu, M., Yurttas, P., Perlin, J., Herr, J. and Coonrod, S., 2007. Proteomic profiling of murine oocyte maturation. Molecular reproduction and development, 74(5): 608-616.

Wang, S., Kou, Z., Jing, Z., Zhang, Y., Guo, X., Dong, M., Wilmut, I. and Gao, S., 2010. Proteome of mouse oocytes at different developmental stages. Proceedings of the National Academy of Sciences, 107(41): 17639-17644.

Zhang, P., Ni, X., Guo, Y., Guo, X., Wang, Y., Zhou, Z., Huo, R. and Sha, J., 2009.Proteomic-based identification of maternal proteins in mature mouse oocytes. BMC genomics, 10(1): 1-11.

\section{How to cite this article:}

Raghvendra Kumar and Prabhat Palta. 2020. Mammalian Oocyte Quality in Relation to Proteome at Different Stages of Development: An Update. Int.J.Curr.Microbiol.App.Sci. 9(10): 3300-3405. doi: https://doi.org/10.20546/ijcmas.2020.910.393 